\title{
Sum Rate Maximizing Superposition Coding Scheme for a Two-User Wireless Relay System
}

\section{$\operatorname{AUTHOR}(\mathrm{S}):$}

Kaneko, Megumi; Hayashi, Kazunori; Sakai, Hideaki

\section{CITATION:}

Kaneko, Megumi ...[et al]. Sum Rate Maximizing Superposition Coding Scheme for a TwoUser Wireless Relay System. IEEE Communications Letters 2011, 15(4): 428-430

\section{ISSUE DATE:}

2011-04

URL:

http://hdl.handle.net/2433/141792

\section{RIGHT:}

(C) 2011 IEEE. Personal use of this material is permitted. Permission from IEEE must be obtained for all other uses, in any current or future media, including reprinting/republishing this material for advertising or promotional purposes, creating new collective works, for resale or redistribution to servers or lists, or reuse of any copyrighted component of this work in other works.; この論文は出版社版でありません。引用の際には出版社版をご確認ざ利用ください。; This is not the published version. Please cite only the published version. 


\title{
Sum Rate Maximizing Superposition Coding Scheme for a Two-User Wireless Relay System
}

\author{
Megumi Kaneko, Kazunori Hayashi and Hideaki Sakai \\ Graduate School of Informatics, Kyoto University, Yoshida Honmachi Sakyo-ku, Kyoto, 606-8501, Japan \\ Email: \{meg,kazunori,hsakai@i.kyoto-u.ac.jp\}
}

\begin{abstract}
A wireless system is considered where users are served in Downlink (DL) by one Base Station (BS) and one Relay Station (RS). A scheme based on Superposition Coding (SC) is proposed, where two users are served by three superposed layers that take advantage of the three available links for maximizing sum rate. With the derived optimal allocation parameters, our scheme outperforms benchmark schemes for sum-rate and fairness, making it very efficient for scheduling multiple users.
\end{abstract}

\section{INTRODUCTION}

Many works in wireless relay systems have shown the efficiency of cooperative diversity, where the broadcast nature of the wireless medium is taken advantage of by combining the different received signals at the destination. Recently, [1] introduced a relaying scheme based on Superposition Coding (SC) that increases spectral efficiency, for a single user system with a Base Station (BS) and a Relay Station (RS) based on Decode-and-Forward (DF) half-duplexing. SC was first introduced in broadcast channels, where several nodes are served by the BS [2]. In the Single-User SC (SU-SC) scheme of [1], BS creates two messages (basic and superposed), both destined to the single user, and transmits them in Step 1. In Step 2, after decoding both messages, RS only forwards the superposed one, which is also used to retrieve the basic message from the signal received in Step 1. This scheme outperforms the Single-User Multi-Hop ( $S U-M H)$ scheme where the message is sent via the relayed link with optimal time division, and the cooperative DF scheme [1]. A two-user SC-based scheme was proposed in [3], where the signals of the two users are superposed but only the message to one user is forwarded.

We aim at devising new scheduling algorithms in a multiuser relay system that improve throughput. We first focus on the Downlink (DL) of a two-user relay system, or Relay Broadcast Channel (RBC), as users $\mathrm{MS}_{1}$ and $\mathrm{MS}_{2}$ are served by a BS and a RS as in Fig. 1. We assume two types of relayed users, $\mathrm{MS}_{1}$ with a direct and relayed link, and $\mathrm{MS}_{2}$ with only a relayed link. The achievable rate region of $R B C$ has been studied in [4] for discrete memoryless channels and in [5] for Gaussian channels and half-duplex RS, where two equally divided orthogonal subchannels are required to partition BS and RS transmissions and no resource optimization is performed among them. Our goal is not to investigate the $R B C$ capacity region, but to design allocation

This work was supported by the Grant-in-Aid for JSPS Fellow no. 204205 and for Scientific Research no. 21760289 from the Ministry of Education, Science, Sports, and Culture of Japan. schemes that enhance existing schedulers. We propose the 3$S C$ Layer scheme, where the signals of the two users are superposed into 3 layers: 2 layers for $\mathrm{MS}_{1}$ and one for $\mathrm{MS}_{2}$. Instead of relaying only $\mathrm{MS}_{2}$ 's data as in [3], the relayed link is shared between the users by using the RS-( $\left.\mathrm{MS}_{1}, \mathrm{MS}_{2}\right)$ link as a broadcast channel. Sum-rate optimization is considered without fairness constraints. Despite being suboptimal, our scheme achieves a much larger sum rate compared to the $R B C$ achievable sum rate in [5], due to resource optimization. Interestingly, unlike in broadcast channels where the best link user alone is served for maximizing sum-rate, we show that serving both users in RBC can increase sum-rate. Even with a large number of users, our scheme outperforms benchmark ones, while improving fairness.

\section{SYSTEM MODEL}

We consider the system in Fig. 1. In step 1, BS transmits a vector $\mathbf{x}$ of $M$ complex baseband symbols $x[m], m \in$ $\{1, \ldots, M\}$. The received signals at $\mathrm{RS}$ and $\mathrm{MS}_{1}$ are $\mathbf{y}_{\mathrm{R}}=$ $h_{\mathrm{R}} \mathbf{x}+\mathbf{z}_{\mathrm{R}}$ and $\mathbf{y}_{\mathrm{D} 1}=h_{\mathrm{D} 1} \mathbf{x}+\mathbf{z}_{1}$. In step 2, RS transmits a vector $\mathrm{x}_{\mathrm{R}}$ of $M_{\mathrm{R}}$ complex baseband symbols. The received signal at $\mathrm{MS}_{i}, i \in\{1,2\}$ is $\mathbf{y}_{\mathrm{R} i}=h_{\mathrm{R} i} \mathbf{x}_{\mathrm{R}}+\mathbf{z}_{i}^{\prime} . h_{\mathrm{R}}, h_{\mathrm{D} 1}$, $h_{\mathrm{R} i}$ are the complex channel coefficients of the BS-RS, BS$\mathrm{MS}_{1}$ and RS-MS ${ }_{i}$ channels. $\mathbf{z}_{\mathrm{R}}, \mathbf{z}_{1}, \mathbf{z}_{i}^{\prime}$ are vectors of complex additive white Gaussian noise with a circular-symmetric distribution $\mathcal{C N}\left(\mathbf{0}, \sigma^{2} \mathbf{I}\right)$. Transmitted symbols satisfy $\mathrm{E}\{x[m]\}=0$ and $\mathrm{E}\left\{|x[m]|^{2}\right\}=1$. Link SNRs are given by $\gamma_{S}=\frac{\left|h_{S}\right|^{2}}{\sigma^{2}}$, $S \in\{\mathrm{D} 1, \mathrm{R}, \mathrm{R} 1, \mathrm{R} 2\}$. The capacity of each link is given by $C\left(\gamma_{S}\right)=\log _{2}\left(1+\gamma_{S}\right)[$ bits/s] for a bandwidth of $1 \mathrm{~Hz}$.

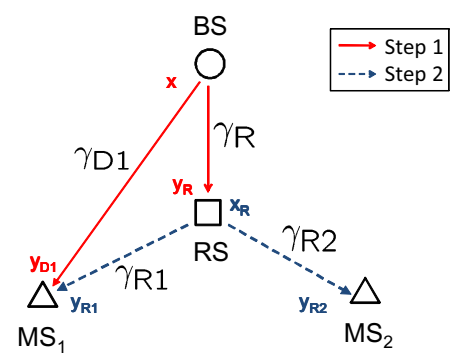

Fig. 1. System Model and Steps of the Proposed Scheme

In the analysis, we assume constant link SNRs ordered as

$$
\gamma_{\mathrm{D} 1}<\gamma_{\mathrm{R} 1}<\gamma_{\mathrm{R} 2}<\gamma_{\mathrm{R}}
$$

In the scenario of multiple users and random channel fading, our scheduler will select user pairs satisfying (1). 
TABLE I

DESCRIPTION OF THE STEPS IN THE PROPOSED SCHEME
TABLE II

CONSTRAINTS ON THE RATES IN THE PROPOSED SCHEME

\begin{tabular}{|c|c|c|c|c|}
\hline Step 1 & $\begin{array}{l}\text { BS sends } \mathbf{x}=\sqrt{\alpha_{\mathrm{b} 1}} \mathbf{x}_{\mathrm{b} 1}+\sqrt{\alpha_{\mathrm{s} 1}} \mathbf{x}_{\mathrm{s} 1}+\sqrt{\alpha_{2}} \mathbf{x}_{2} \\
\text { with } \alpha_{\mathrm{b} 1}+\alpha_{\mathrm{s} 1}+\alpha_{2}=1 \\
\mathrm{RS} \text { receives } \mathbf{y}_{\mathrm{R}}=h_{\mathrm{R}}\left(\sqrt{\alpha_{\mathrm{b} 1}} \mathbf{x}_{\mathrm{b} 1}+\sqrt{\alpha_{\mathrm{s} 1}} \mathbf{x}_{\mathrm{s} 1}+\sqrt{\alpha_{2}} \mathbf{x}_{2}\right)+\mathbf{z}_{\mathrm{R}} \\
\mathrm{MS}_{1} \text { receives } \mathbf{y}_{\mathrm{D} 1}=h_{\mathrm{D} 1}\left(\sqrt{\alpha_{\mathrm{b} 1}} \mathbf{x}_{\mathrm{b} 1}+\sqrt{\alpha_{\mathrm{s} 1}} \mathbf{x}_{\mathrm{s} 1}+\sqrt{\alpha_{2}} \mathbf{x}_{2}\right)+\mathbf{z}_{1}\end{array}$ & Step 1 & $\left.\begin{array}{l}R_{\mathrm{b} 1} \leq C \\
R_{\mathrm{s} 1} \leq C\end{array}\right\}$ & $\begin{array}{l}\left.\frac{\alpha_{\mathrm{b} 1} \gamma_{\mathrm{R}}}{1+\left(\alpha_{\mathrm{s} 1}+\alpha_{2}\right) \gamma_{\mathrm{R}}}\right) \stackrel{\text { def }}{=} R_{\mathrm{b} 1}^{\mathrm{A}} \\
\left.\frac{\alpha_{\mathrm{s} 1} \gamma_{\mathrm{R}}}{1+\alpha_{2} \gamma_{\mathrm{R}}}\right), \quad R_{2} \leq C\left(\alpha_{2} \gamma_{\mathrm{R}}\right)\end{array}$ \\
\hline Step 2 & $\begin{array}{l}\mathrm{RS} \text { sends } \mathbf{x}_{\mathrm{R}}=\sqrt{1-\beta} \mathbf{x}_{\mathrm{R} 1}+\sqrt{\beta} \mathbf{x}_{\mathrm{R} 2} \\
\mathrm{MS}_{1} \text { receives } \mathbf{y}_{\mathrm{R} 1}=h_{\mathrm{R} 1}\left(\sqrt{1-\beta} \mathbf{x}_{\mathrm{R} 1}+\sqrt{\beta} \mathbf{x}_{\mathrm{R} 2}\right)+\mathbf{z}^{\prime}{ }_{1} \\
\mathrm{MS}_{1} \text { decodes } \mathbf{x}_{\mathrm{s} 1} \rightarrow \mathbf{y}^{\prime}{ }_{\mathrm{D} 1}=h_{\mathrm{D} 1}\left(\sqrt{\alpha_{\mathrm{b} 1}} \mathbf{x}_{\mathrm{b} 1}+\sqrt{\alpha_{2}} \mathbf{x}_{2}\right)+\mathbf{z}_{1} \\
\mathrm{MS}_{2} \text { receives } \mathbf{y}_{\mathrm{R} 2}=h_{\mathrm{R} 2}\left(\sqrt{1-\beta} \mathbf{x}_{\mathrm{R} 1}+\sqrt{\beta} \mathbf{x}_{\mathrm{R} 2}\right)+\mathbf{z}^{\prime}{ }_{2} \\
\mathrm{MS}_{2} \text { decodes } \mathbf{x}_{\mathrm{R} 1} \rightarrow \mathbf{y}^{\prime}{ }_{\mathrm{R} 2}=h_{\mathrm{R} 2} \sqrt{\beta} \mathbf{x}_{\mathrm{R} 2}+\mathbf{z}^{\prime}{ }_{2}\end{array}$ & Step 2 & $\begin{array}{l}R_{\mathrm{R} 1} \leq C \\
R_{\mathrm{b} 1} \leq C\end{array}$ & $\begin{array}{l}\left(\frac{1+\alpha_{2} \gamma_{\mathrm{R}}}{1+\beta \gamma_{\mathrm{R} 1}}\right. \\
\left.\frac{\alpha_{\mathrm{b} 1} \gamma_{\mathrm{D} 1}}{1+\alpha_{2} \gamma_{\mathrm{D} 1}}\right) \stackrel{\text { def }}{=} R_{\mathrm{b} 1}^{\mathrm{B}}, \quad R_{\mathrm{R} 2}\end{array}$ \\
\hline
\end{tabular}

\section{Proposed Scheme: 3-SC Layer Scheme}

We describe the steps of the proposed scheme, while the equations for each signal are given in Table I.

Step 1: The BS sends message $\mathrm{x}$ composed of 3 superposed messages: $\mathbf{x}_{\mathrm{b} 1}, \mathrm{x}_{\mathrm{s} 1}$ for $\mathrm{MS}_{1}$ and $\mathrm{x}_{2}$ for $\mathrm{MS}_{2}$ with power allocation ratios $\alpha_{\mathrm{b} 1}, \alpha_{\mathrm{s} 1}$ and $\alpha_{2} \in[0,1]$, respectively. We refer to $\mathbf{x}_{\mathrm{b} 1}$ as the basic message for $\mathrm{MS}_{1}$ and $\mathrm{x}_{\mathrm{s} 1}$ as the superposed message for $\mathrm{MS}_{1}$. The sum of power allocation ratios is equal to one. Then, the RS receives $\mathbf{y}_{R}$ from which it decodes each message one by one, treating the other messages as noise by Successive Interference Cancelation (SIC). The decoding order follows the order of increasing link SNRs $\gamma_{\mathrm{D} 1}<\gamma_{\mathrm{R} 1}<\gamma_{\mathrm{R} 2}$ as in [6], i.e., $\mathbf{x}_{\mathrm{b} 1} \rightarrow \mathbf{x}_{\mathrm{s} 1} \rightarrow \mathbf{x}_{2}$ at RS, shown to be very effective by our numerical results. On the other hand, $\mathrm{MS}_{1}$ receives $\mathbf{y}_{\mathrm{D} 1}$ and keeps it in memory.

Step 2: RS sends message $x_{R}$ which superposes $x_{R 1}$ and $\mathbf{x}_{\mathrm{R} 2}$, the decoded and remodulated signals of $\mathbf{x}_{\mathrm{s} 1}$ and $\mathbf{x}_{2}$, respectively, with the power allocation ratio $\beta \in[0,1]$. Then, $\mathrm{MS}_{1}$ receives $\mathbf{y}_{\mathrm{R} 1}$ from which it decodes $\mathbf{x}_{\mathrm{R} 1}\left(\mathbf{x}_{s 1}\right)$, treating $\sqrt{\beta} \mathbf{x}_{\mathrm{R} 2}$ as noise (as $\left.\gamma_{\mathrm{R} 1}<\gamma_{\mathrm{R} 2}\right)$. From $\mathbf{y}_{\mathrm{D} 1}$ received in Step $1, \mathrm{MS}_{1}$ cancels $\mathbf{x}_{\mathrm{s} 1}$, giving $\mathbf{y}_{\mathrm{D} 1}^{\prime}$. Finally, $\mathrm{MS}_{1}$ decodes $\mathrm{x}_{\mathrm{b} 1}$ with noise $\sqrt{\alpha_{2}} \mathbf{x}_{2}$. In the same way, $\mathrm{MS}_{2}$ receives $\mathbf{y}_{\mathrm{R} 2}$, from which it decodes $\mathbf{x}_{\mathrm{R} 1}$. Canceling $\mathbf{x}_{\mathrm{R} 1}$ from $\mathbf{y}_{\mathrm{R} 2}, \mathrm{MS}_{2}$ gets $\mathbf{y}^{\prime}{ }_{\mathrm{R} 2}$, from which $\mathbf{x}_{\mathrm{R} 2}\left(\mathbf{x}_{2}\right)$ is decoded.

We denote by $R_{\mathrm{b} 1}, R_{\mathrm{s} 1}$ the rates of the basic and superposed messages for $\mathrm{MS}_{1}$; by $R_{\mathrm{R} i}, i=\{1,2\}$, the rate of the relayed messages $\mathbf{x}_{\mathrm{R} i}$. The constraints on these rates are given in Table II. In Step 1, the constraints ensure that RS can decode $\mathbf{x}_{\mathrm{b} 1}, \mathbf{x}_{\mathrm{s} 1}, \mathbf{x}_{2}$ respectively. In Step 2, the first two constraints ensure that $\mathrm{MS}_{1}$ decodes $\mathbf{x}_{\mathrm{R} 1}$ and $\mathbf{x}_{\mathrm{b} 1}$, while the first and last constraints ensure that $\mathrm{MS}_{2}$ decodes $\mathbf{x}_{\mathrm{R} 1}$ (as $\gamma_{\mathrm{R} 1}<\gamma_{\mathrm{R} 2}$ ) and $\mathrm{x}_{\mathrm{R} 2}$. We define $R_{\mathrm{b} 1}^{\mathrm{A}}, R_{\mathrm{b} 1}^{\mathrm{B}}$ to denote the two constraints on $R_{\mathrm{b} 1}$ to be satisfied, so $R_{\mathrm{b} 1}=\min \left(R_{\mathrm{b} 1}^{\mathrm{A}}, R_{\mathrm{b} 1}^{\mathrm{B}}\right)$. All the other rates are equal to their capacity expressions in Table II.

BS transmits $M\left(R_{\mathrm{b} 1}+R_{\mathrm{s} 1}+R_{2}\right)$ bits in Step 1. In Step 2, RS forwards $M\left(R_{\mathrm{s} 1}+R_{2}\right)$ bits. The transmission time $M_{\mathrm{R}}$ at Step 2 is the larger one between the times to $\mathrm{MS}_{1}$ and $\mathrm{MS}_{2}$, i.e., $M_{1}=M I_{1}$ with $I_{1}=\frac{R_{\mathrm{s} 1}}{R_{\mathrm{R} 1}}$ and $M_{2}=M I_{2}$ with $I_{2}=\frac{R_{2}}{R_{\mathrm{R} 2}}$. With the constraints in Table II, the sum rate becomes

$$
R_{3 \mathrm{~L}}=\frac{\min \left(R_{\mathrm{b} 1}^{\mathrm{A}}, R_{\mathrm{b} 1}^{\mathrm{B}}\right)+R_{\mathrm{s} 1}+R_{2}}{1+\max \left(I_{1}, I_{2}\right)} .
$$

Next, we determine the power allocation ratios that maximize the sum rate. Defining $\alpha_{\mathrm{s} 1}^{*}=\frac{1}{\gamma_{\mathrm{D} 1}}-\frac{1}{\gamma_{\mathrm{R}}}$, we distinguish two cases (we assume $\alpha_{\mathrm{s} 1}^{*} \leq 1$; otherwise multi-hop transmission may be used for $\mathrm{MS}_{1}$ as in [1]).

Case 1: $R_{\mathrm{b} 1}^{\mathrm{A}} \leq R_{\mathrm{b} 1}^{\mathrm{B}} \Longleftrightarrow \alpha_{\mathrm{s} 1} \geq \alpha_{\mathrm{s} 1}^{*}$, we have $R_{3 \mathrm{~L}}=$ $\frac{\log _{2}\left(1+\gamma_{\mathrm{R}}\right)}{1+\max \left(I_{1}^{(1)}, I_{2}^{(1)}\right)} \stackrel{\text { def }}{=} R_{3 \mathrm{~L}}^{(1)}$ where

$$
I_{1}^{(1)}=\frac{\log _{2}\left(\frac{1+\left(\alpha_{\mathrm{s} 1}+\alpha_{2}\right) \gamma_{\mathrm{R}}}{1+\alpha_{2} \gamma_{\mathrm{R}}}\right)}{\log _{2}\left(\frac{1+\gamma_{\mathrm{R} 1}}{1+\beta \gamma_{\mathrm{R} 1}}\right)}, I_{2}^{(1)}=\frac{\log _{2}\left(1+\alpha_{2} \gamma_{\mathrm{R}}\right)}{\log _{2}\left(1+\beta \gamma_{\mathrm{R} 2}\right)} .
$$

$R_{3 \mathrm{~L}}$ increases as $\alpha_{\mathrm{s} 1}$ decreases, so we set $\alpha_{\mathrm{s} 1}=\alpha_{\mathrm{s} 1}^{*}$.

Case 2: $R_{\mathrm{b} 1}^{\mathrm{B}} \leq R_{\mathrm{b} 1}^{\mathrm{A}} \Longleftrightarrow \alpha_{\mathrm{s} 1} \leq \alpha_{\mathrm{s} 1}^{*}$, Here, by derivation of $R_{3 \mathrm{~L}}$ in function of $\alpha_{\mathrm{b} 1}$, we find that it is a decreasing function of $\alpha_{\mathrm{b} 1}$, hence we set $\alpha_{\mathrm{b} 1}=0$. Thus, $\alpha_{\mathrm{s} 1}+\alpha_{2}=1$, and $R_{3 \mathrm{~L}}=\frac{\log _{2}\left(1+\gamma_{\mathrm{R}}\right)}{1+\max \left(I_{1}^{(2)}, I_{2}^{(2)}\right)} \stackrel{\text { def }}{=} R_{3 \mathrm{~L}}^{(2)}$, where

$$
I_{1}^{(2)}=\frac{\log _{2}\left(\frac{1+\gamma_{\mathrm{R}}}{1+\alpha_{2} \gamma_{\mathrm{R}}}\right)}{\log _{2}\left(\frac{1+\gamma_{\mathrm{R} 1}}{1+\beta \gamma_{\mathrm{R} 1}}\right)}, \quad I_{2}^{(2)}=\frac{\log _{2}\left(1+\alpha_{2} \gamma_{\mathrm{R}}\right)}{\log _{2}\left(1+\beta \gamma_{\mathrm{R} 2}\right)} .
$$

In both cases, maximizing sum rate reduces to the problem of minimizing the transmission time $M_{\mathrm{R}}$ of Step 2. Thus, we solve the following problem for each Case $i \in\{1,2\}$,

$$
\begin{aligned}
& \min _{\alpha_{2}, \beta} \max \left(I_{1}^{(i)}, I_{2}^{(i)}\right), \\
& \text { s.t. } \alpha_{\mathrm{b} 1}+\alpha_{\mathrm{s} 1}+\alpha_{2}=1, \quad 0 \leq \beta \leq 1 .
\end{aligned}
$$

We denote $\max \left(I_{1}^{(i)}, I_{2}^{(i)}\right)$ by $I_{\mathrm{R}}^{(i)}\left(\alpha_{2}, \beta\right)$ as it has only 2 variables $\left(\alpha_{2}, \beta\right)$. That is, in Case 1 , we have $\alpha_{\mathrm{s} 1}=\alpha_{\mathrm{s} 1}^{*}$ and $\alpha_{\mathrm{b} 1}$ is fixed by constraint (3). In Case 2, $\alpha_{\mathrm{s} 1}=1-\alpha_{2}$ and $\alpha_{\mathrm{b} 1}=0$. The global solution $\left(\alpha_{2}^{*}, \beta^{*}\right)$ is given by the minimum $I_{\mathrm{R}}^{(i)}$ between the 2 cases. For boundary values, $\alpha_{2}=0$ implies $\beta=0$ since there is nothing to forward to $\mathrm{MS}_{2}$. Thus, $I_{\mathrm{R}}^{(1)}(0,0)=\frac{\log _{2}\left(1+\alpha_{\mathrm{s} 1}^{*} \gamma_{\mathrm{R}}\right)}{\log _{2}\left(1+\gamma_{\mathrm{R}}\right)}$. If $\alpha_{2}=1$ (only in Case 2), then $\beta=1$ as nothing is forwarded to $\mathrm{MS}_{1}$, so $I_{\mathrm{R}}^{(2)}(1,1)=\frac{\log _{2}\left(1+\gamma_{\mathrm{R}}\right)}{\log _{2}\left(1+\gamma_{\mathrm{R} 2}\right)}$. Removing these cases, the domain of (3) is $0<\alpha_{2}<1,0<\beta<1$, i.e., $I_{1}>0$ and $I_{2}>0$.

We show that for any given $\alpha_{2}$ in $] 0,1\left[, I_{\mathrm{R}}\right.$ is minimized for $\beta$ in ] 0,1 , such that $I_{1}=I_{2}$, as $I_{1}$ and $I_{2}$ are monotonically increasing and decreasing functions of $\beta$, respectively. We define $f(\beta)=R_{\mathrm{s} 1} R_{\mathrm{R} 2}-R_{2} R_{\mathrm{R} 1}$. For any $\alpha_{2}$ in ]0,1[, there exists a unique $\beta=\beta_{2}$ such that $f\left(\beta_{2}\right)=0$ which is equivalent to $I_{1}=I_{2}$, as $f(0)<0, f(1)>0$ and $\frac{\partial f}{\partial \beta}>0$. This gives a bijection from $\alpha_{2}$ to $\beta_{2}$, so we just need to find the optimum $\hat{\alpha}_{2}$ over the reduced domain in each case, if it exists, and then compare $I_{\mathrm{R}}$ for $\hat{\alpha}_{2}$ with the boundary values, to determine the minimum over the whole domain. If it exists, $\hat{\alpha}_{2}$ is found by setting the derivative of $I_{1}$ and $I_{2}$ with respect 


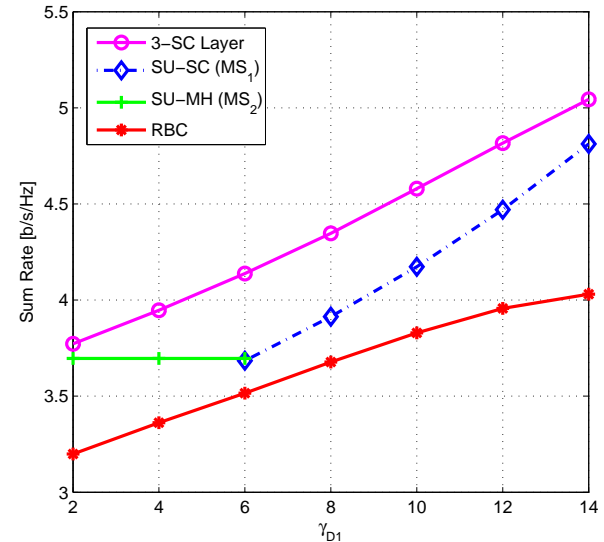

Fig. 2. Achievable sum rate for different schemes $\left(\gamma_{\mathrm{D} 1}=2-14 \mathrm{~dB}, \gamma_{\mathrm{R} 1}=15 \mathrm{~dB}\right.$, $\left.\gamma_{\mathrm{R} 2}=20 \mathrm{~dB}, \gamma_{\mathrm{R}}=25 \mathrm{~dB}\right)$

to $\alpha_{2}$ to zero (with $I_{1}=I_{2}$ from $\beta_{2}$ ), which gives in Case 1,

$$
\begin{aligned}
& \frac{\gamma_{\mathrm{R}}}{1+\alpha_{2} \gamma_{\mathrm{R}}} C\left(\beta_{2} \gamma_{\mathrm{R} 2}\right)-\frac{\gamma_{\mathrm{R} 2}}{1+\beta_{2} \gamma_{\mathrm{R} 2}} \frac{\partial \beta_{2}}{\partial \alpha_{2}} C\left(\alpha_{2} \gamma_{\mathrm{R}}\right)=0, \\
& -\frac{\alpha_{\mathrm{s} 1}^{*} \gamma_{\mathrm{R}}^{2}}{\left(1+\alpha_{2} \gamma_{\mathrm{R}}\right)\left(1+\left(\alpha_{\mathrm{s} 1}^{*}+\alpha_{2}\right) \gamma_{\mathrm{R}}\right)} \log _{2}\left(\frac{1+\gamma_{\mathrm{R} 1}}{1+\beta_{2} \gamma_{\mathrm{R} 1}}\right) \\
& +\frac{\gamma_{\mathrm{R} 1}}{1+\beta_{2} \gamma_{\mathrm{R} 1}} \frac{\partial \beta_{2}}{\partial \alpha_{2}} \log _{2}\left(\frac{1+\left(\alpha_{\mathrm{s} 1}^{*}+\alpha_{2}\right) \gamma_{\mathrm{R}}}{1+\alpha_{2} \gamma_{\mathrm{R}}}\right)=0 .
\end{aligned}
$$

Eliminating $\frac{\partial \beta_{2}}{\partial \alpha_{2}}$ and using the equation $f\left(\beta_{2}\right)=0$, we obtain

$$
\hat{\alpha}_{2}^{(1)}=\left(\frac{1}{\gamma_{\mathrm{D} 1}}-\frac{1}{\gamma_{\mathrm{R}}}\right) \frac{\gamma_{\mathrm{R} 2}}{\gamma_{\mathrm{R} 1}} \frac{1+\beta_{2}^{*} \gamma_{\mathrm{R} 1}}{1+\beta_{2}^{*} \gamma_{\mathrm{R} 2}}-\frac{1}{\gamma_{\mathrm{D} 1}},
$$

where $\beta_{2}^{*}$ is the value of $\beta_{2}$ for $\hat{\alpha}_{2}^{(1)}$. By inserting Eq. (4) into $f(\beta)=0$, we get $\beta_{2}^{*}$ numerically by Newton's method, as there exists a unique $\beta_{2}$ for any $\alpha_{2}$, so in particular for $\hat{\alpha}_{2}^{(1)}$.

In Case 2, we find $\hat{\alpha}_{2}^{(2)}=\emptyset$. Finally, the global minimum $\left(\alpha_{2}^{*}, \beta^{*}\right)$ is determined comparing $I_{\mathrm{R}}^{(1)}(0,0), I_{\mathrm{R}}^{(2)}(1,1)$ and $I_{\mathrm{R}}^{(1)}\left(\hat{\alpha}_{2}^{(1)}, \beta_{2}^{*}\right)$.

\section{Numerical Results}

The sum-rates of our 3-SC Layer and benchmark schemes are plotted in Fig. 2 for two users and specified SNR values. The benchmark scheme from [1] allocates the user with the highest rate for a given set of SNRs, which is achieved by $\mathrm{MS}_{2}$ with $S U-M H$ for $2 \mathrm{~dB} \leq \gamma_{\mathrm{D} 1}<6 \mathrm{~dB}$, and by $\mathrm{MS}_{1}$ with $S U$ $S C$ for $\gamma_{\mathrm{D} 1} \geq 6 \mathrm{~dB}$. Fig. 2 shows that this benchmark scheme is largely outperformed by 3-SC Layer for all $\gamma_{\mathrm{D} 1}$. Note that the scheme in [3] under sum rate maximization achieves the same rate as $S U$-SC, as the superposed message is sent at rate $C\left(\gamma_{\mathrm{R} 1}\right)$. Finally, our scheme improves over the sum rate of the $R B C$ in [5] that requires two equally divided orthogonal subchannels, stressing the benefit of our resource optimization.

Next, we evaluate the impact of our scheme for multi-user scheduling. We assume 20 users, half of them supported by direct and relayed links (type 1), and half without a direct link (type 2). The BS-RS link SNR is fixed to $30 \mathrm{~dB}$, but other channel SNRs are generated by the exponential distribution with mean $\bar{\gamma}_{\mathrm{D} 1}=5 \mathrm{~dB}$ for direct links of type 1 users and $\bar{\gamma}_{\mathrm{R} 1}=\bar{\gamma}_{\mathrm{R} 2}=15 \mathrm{~dB}$ for relayed links of both types of users. Benchmark schedulers are based on the Max SINR scheme of [7], where the user with the highest link quality is allocated all resources in each channel, a strategy commonly taken for throughput maximization in relayed networks with multiple users. Equivalently, benchmark Schedulers 2 and 3 allocate the user with highest rate among single user schemes. In Scheduler 3, only Direct transmission or $S U-M H$ is available for $\mathrm{MS}_{1}{ }^{1}$ and $S U-M H$ for $\mathrm{MS}_{2}$. In Scheduler 2, additionally, $S U$-SC may be used for $\mathrm{MS}_{1}$. Scheduler 1 includes our 3$S C$ Layer scheme, which is applied to all user pairs of type 1 and 2, and the pair with the best sum-rate is scheduled if it outperforms the best single user rate. Table III shows the sum-rate in $[\mathrm{b} / \mathrm{s} / \mathrm{Hz}]$ of each scheduler and their fairness measured every ten frames by Jain's Index [7], as well as the percentage that each scheme (Direct, SU-MH, SU-SC, 3SC$L)$ achieved the best sum-rate. Scheduler 1 outperforms both reference ones, owing to 3-SC Layer which achieves the best sum-rate in $82 \%$ of the cases. Comparing Schedulers 2 and 3, we see that $S U$-SC from [1] contributes to enhance sum-rate but decreases fairness as only type 1 user rates are improved. However, Scheduler 1 outperforms both sum-rate and fairness of Schedulers 2 and 3. The ability to improve two opposite measures such as system throughput and fairness makes our scheme very appealing for, e.g., best-effort traffics which are not fairness-constrained although fairness is a critical measure.

TABLE III

SCheduling Performance With 20 USERS

\begin{tabular}{|c|c|c|c|c|c|c|}
\hline Scheduler & Sum-rate & Fairness & Direct & SU-MH & SU-SC & 3SC-L \\
\hline 1 & 4.7 & 0.44 & $0 \%$ & $0 \%$ & $18 \%$ & $82 \%$ \\
\hline 2 & 4.4 & 0.29 & $1 \%$ & $13 \%$ & $86 \%$ & - \\
\hline 3 & 4.0 & 0.39 & $10 \%$ & $90 \%$ & - & - \\
\hline
\end{tabular}

\section{CONCLUSion}

We have proposed the 3-SC Layer scheme for allocating DL resources in a two-user wireless relay system. By splitting the messages to the two users into three superposed layers, this scheme takes advantage of the relayed links as in a broadcast channel. With the derived optimal power allocation under sum-rate maximization, our scheme outperformed benchmark schemes and the achievable sum rate for $R B C$ in [5]. The benefits in sum-rate and fairness of the proposed scheme were also shown for a large number users, which makes it very promising for multi-user scheduling in general relay systems.

\section{REFERENCES}

[1] P. Popovski and E. de Carvalho, "Improving the Rates in Wireless Relay Systems through Superposition Coding," IEEE Trans. Wireless Comm., vol. 7 , no. 12 , pp. 4831-4836, December 2008.

[2] T.M. Cover and J.A. Thomas, Elements of Information Theory. John Wiley \& Sons Inc., 2006.

[3] P. Popovski, et al., "Method and apparatus for transmitting and receiving data using multi-user superposition coding in a wireless relay system," US Patent No. US2008/0227388A1, Sept. 2008.

[4] G. Kramer, M. Gastpar and P. Gupta, "Cooperative Strategies and Capacity Theorems for Relay Networks," IEEE Trans. on Info. Theory, vol. 51, no. 9, pp. 3037-3063, September 2005.

[5] Y. Liang and V. Veeravalli, "The Impact of Relaying on the Capacity of Broadcast Channels," in IEEE ISIT, Chicago, USA, June 2004.

[6] D. Tse and P. Viswanath, Fundamentals of Wireless Communication. Cambridge, UK: Cambridge University Press, 2005.

[7] M. Salem et al., "An Overview of Radio Resource Management in RelayEnhanced OFDMA-Based Networks," IEEE Comm. Surveys \& Tutorials, vol. 12, no. 3, pp. 422-438, Third Quarter 2010.

\footnotetext{
${ }^{1}$ Cooperative diversity could be used for $\mathrm{MS}_{1}$, but such a scheduler achieves lower fairness than Scheduler 3 and lower sum-rate than Scheduler 2.
} 